\title{
Fault Tolerant Consensus of Multi-Agent Systems with Linear Dynamics
}

\author{
Jianzhen Li \\ School of Electronics and Information, Jiangsu University of Science and Technology, Zhenjiang, Jiangsu 212003, China \\ Correspondence should be addressed to Jianzhen Li; jzlijust@gmail.com
}

Received 14 November 2013; Accepted 7 December 2013

Academic Editor: Hao Shen

Copyright ( 2013 Jianzhen Li. This is an open access article distributed under the Creative Commons Attribution License, which permits unrestricted use, distribution, and reproduction in any medium, provided the original work is properly cited.

\begin{abstract}
This paper deals with the consensus problem of linear multi-agent systems with actuator faults. A fault estimator based consensus protocol is provided, together with a convergence analysis. It is shown that the consensus errors of all agents will converge to a small set around the origin, if parameters in the consensus protocol are properly chosen. A numerical example is given to illustrate the effectiveness of the proposed protocol.
\end{abstract}

\section{Introduction}

Recently, distributed consensus problems for multi-agent systems have become a hot research area in the control theory community [1-12]. This is partly because of their widespread applications in areas such as robots, flocking, unmanned air vehicles, sensor fusion, and microgrids (see [13-18] and the references therein). For multi-agent systems, consensus means the group of agents asymptotically agree on certain quantities of interest that depends on the states of all agents [19]. In the research of multi-agent systems, the main challenge is how to design simple control rule for simple agents to achieve a prescribed group behavior.

Fault detection and fault accommodation are very important problems for control systems [20-22]. The fault detection and the fault tolerant consensus problems of multi-agent systems have attracted attention of researchers in the last few years. In [23], the fault detection problem was considered for discrete-time multi-agent systems with first-order dynamics, while the continuous-time second-order multi-agent systems were considered in [24]. The fault tolerant consensus problem for first-order multi-agent systems was investigated in [25], under the assumption that the faults are detected in time. The resilient consensus problem was considered in [26], for multi-agent systems with adversary agents. A consensus protocol is provided; under which consensus can be achieved, if the number of adversary agents satisfies a certain condition related to the degree of the communication graph.
The fault tolerant consensus problem for high-order linear multi-agent systems has not been considered in the literature, which motivated the work in this paper. In this paper, consensus problems will be considered for linear multi-agent systems with actuator faults. A fault estimator is provided, based on which a consensus protocol is derived. It is proved that consensus error can converge to a small set around the origin, if parameters in the fault estimator and the consensus protocol are properly chosen. The rest of the paper is organized as follows. Section 2 formulates the fault tolerant consensus problem of the multi-agent systems with linear dynamics. The main results are presented in Section 3. A numerical example is given in Section 4 to illustrate the proposed results and the paper is concluded in Section 5.

Notations. Throughout this paper, matrix $A>0$ means that $A$ is symmetric positive definite. Consider $1_{n}=[1,1, \ldots, 1]^{T} \in$ $R^{n \times 1}$. Similarly, $\mathbf{0}_{n}=[0,0, \ldots, 0]^{T} \in R^{n \times 1}$.

\section{Problem Formulation}

Consider a team of $n$ agents with the following linear dynamics:

$$
\begin{aligned}
& \dot{x}_{i}(t)=A x_{i}(t)+B u_{i}(t)+E f_{i}(t), \\
& y_{i}(t)=C x_{i}(t),
\end{aligned}
$$


where $x_{i}(t) \in \mathbb{R}^{m}$ is the state of agent $i, u_{i}(t) \in \mathbb{R}^{p}$ is the control input, and $y_{i}(t) \in \mathbb{R}^{l}$ is the output. $f_{i}(t) \in \mathbb{R}^{m}$ denotes the actuator fault of agent $i$. If $f_{i}(t) \equiv 0$, then no actuator fault occurs at agent $i$. In this paper, we have the following assumptions.

Assumption 1. Assume that $\left\|f_{i}(t)\right\| \leq f_{0},\left\|\dot{f}_{i}(t)\right\| \leq f_{1}$, for $i=1, \ldots, n$.

Assumption 2. $(A, C)$ is observable, and $C E$ is full rank.

Remark 3. Assumption 2 means that the state of the agent can be constructed from the output, and there is no subsystem decoupled from the faults in the linear description [20]. Under Assumption 2, there exist a symmetric positive definite $P$ and matrices $F$ and $R$ such that

$$
\begin{gathered}
P E=C^{T} R, \\
(A-F C)^{T} P+P(A-F C)<0 .
\end{gathered}
$$

The communication topology among the $n$ agents can be represented by an undirected graph $\mathscr{G}=(\mathscr{V}, \mathscr{E})$, where $\mathscr{V}=\{1, \ldots, n\}$ is the node set and $\mathscr{E} \in \mathscr{V} \times \mathscr{V}$ is the edge set. An edge $(j, i) \in \mathscr{E}$ if agent $i$ and agent $j$ can access information from each other. An undirected path is a sequence of undirected edges of the form $\left(i_{1}, i_{2}\right),\left(i_{2}, i_{3}\right), \ldots$, where $i_{j} \in \mathscr{V}$. An undirected graph is connected if for any $i, j \in \mathscr{V}$ there exists a path between them. The neighbor set $\mathcal{N}_{i}$ of agent $i$ is defined as $\mathcal{N}_{i} \triangleq\{j \mid(j, i) \in \mathscr{E}\}$. The adjacency matrix $\mathscr{A} \triangleq\left[a_{i j}\right] \in \mathbb{R}^{n \times n}$ is defined as $a_{i j}=a_{j i}>0$ if $(j, i) \in \mathscr{E}$ and $a_{i j}=0$ otherwise. The Laplacian matrix $\mathscr{L}$ is defined as $\mathscr{L} \triangleq D-A$, where $D=\operatorname{diag}\left\{\sum_{j=1}^{n} a_{1 j}, \ldots, \sum_{j=1}^{n} a_{n j}\right\}$. It is well known that if the communication topology is connected, then $\mathscr{L}$ has a simple zero eigenvalue and $n-1$ nonnegative eigenvalues $\lambda_{2} \leq \lambda_{3} \leq \cdots \leq \lambda_{n}$.

Definition 4. We say algorithm $u_{i}(t)$ asymptotically solves the consensus problem if $x_{i}(t)-\left(\sum_{j=1}^{N} x_{j}(t)\right) / n \rightarrow 0$ as $t \rightarrow \infty$, for any $i=1, \ldots, n$.

The objective of this paper is to derive a consensus protocol $u_{i}(t)$ under which consensus can be achieved even if some agents are subject to actuator faults.

\section{Main Results}

This section gives the fault tolerant consensus protocol for multi-agent systems described in the last section. Before giving the consensus protocol, we give the fault estimator first.

3.1. Fault Estimator. Consider the following fault estimator, which is motivated by the fault estimator in [20]:

$$
\begin{aligned}
& \dot{\hat{x}}_{i}(t)=A \widehat{x}_{i}(t)+B u_{i}(t)+E \widehat{f}_{i}(t)+F e_{y i}(t), \\
& \dot{\hat{f}}_{i}(t)=\Gamma R^{T} e_{y i}(t)-\sigma \Gamma \widehat{f}_{i}(t), \\
& e_{y i}(t)=C e_{x i}(t),
\end{aligned}
$$

where $\widehat{x}_{i}(t)$ and $\widehat{f}_{i}(t)$ are estimations of $x_{i}(t), y_{i}(t)$, and $f_{i}(t)$, respectively; $e_{x i}(t)=x_{i}(t)-\widehat{x}_{i}(t)$ and $e_{y i}(t)=y_{i}(t)-\widehat{y}_{i}(t)$; matrices $F$ and $R$ are chosen according to (3); matrix $\Gamma>0$ and constant scalar $\sigma>0$ are chosen such that $\sigma-\lambda_{\max }\left(\Gamma^{-1}\right)>$ 0 .

Lemma 5. Define $e_{f i}(t) \triangleq f_{i}(t)-\widehat{f}_{i}(t)$. Under Assumptions 1 and 2, estimator (4) guarantees that $\left(e_{x i}, e_{f i}\right)$ converge exponentially to the following set:

$$
\begin{gathered}
S=\left\{\left(e_{x i}, e_{f i}\right) \mid e_{x i}^{T} \Pi_{1} e_{x i}-\left[\sigma-\lambda_{\max }\left(\Gamma^{-1}\right)\right]\left\|e_{f i}\right\|^{2}\right. \\
\left.\leq \lambda_{\max }\left(\Gamma^{-1}\right) f_{1}^{2}\right\},
\end{gathered}
$$

where $\Pi_{1}=P(A-F C)+(A-F C)^{T} P$.

Proof. The proof is similar to the proof of Theorem 1 in [20] and hence is omitted here.

\subsection{Fault Tolerant Consensus}

Assumption 6. $(A, B)$ is controllable, and $\operatorname{rank}(B, E)=$ $\operatorname{rank}(B)$.

Under Assumption 6, there exists a matrix $B$ such that ( $I-$ $\left.B B^{*}\right) E=0$; that is, $B B^{*} E=E$. Based on estimator (4), we have the following consensus protocol:

$$
u_{i}(t)=K \sum_{j=1}^{n} a_{i j}\left[x_{i}(t)-x_{j}(t)\right]-B^{*} E \widehat{f}_{i}(t),
$$

where $K$ is a parameter matrix to be designed. With (6), system (1) becomes

$$
\begin{aligned}
\dot{x}_{i}(t)= & A x_{i}(t)+B K \sum_{j=1}^{n} a_{i j}\left[x_{i}(t)-x_{j}(t)\right] \\
& -B B^{*} E \widehat{f}_{i}(t)+E f_{i}(t) .
\end{aligned}
$$

Let $e_{i}(t) \triangleq x_{i}(t)-(1 / n) \sum_{j=1}^{n} x_{j}(t)$. It is easy to see that consensus is achieved if $e_{i}(t)=0$, for $i=1, \ldots, n$. From (1) and (6), we have

$$
\begin{gathered}
\dot{e}_{i}(t)=A x_{i}(t)+B K \sum_{j=1}^{n} a_{i j}\left[x_{i}(t)-x_{j}(t)\right] \\
-B B^{*} E \widehat{f}_{i}(t)+E f_{i}(t) \\
-\frac{1}{n} \sum_{j=1}^{n}\left\{A x_{j}(t)+B K \sum_{l=1}^{n} a_{j l}\left[x_{j}(t)-x_{l}(t)\right]\right. \\
\left.-B B^{*} E \widehat{f}_{j}(t)+E f_{j}(t)\right\} .
\end{gathered}
$$


Notice that $-B B^{*} E \widehat{f}_{i}(t)+E f_{i}(t)=E e_{f i}(t), x_{i}(t)-x_{j}(t)=$ $e_{i}(t)-e_{j}(t)$, and $A x_{i}(t)-(1 / n) \sum_{j=1}^{n} A x_{j}(t)=A e_{i}(t)$, and $(8)$ can be rewritten as

$$
\begin{aligned}
\dot{e}_{i}(t)= & A e_{i}(t)+B K \sum_{j=1}^{n} a_{i j}\left[e_{i}(t)-e_{j}(t)\right]+E e_{f i}(t) \\
& -\frac{1}{n} \sum_{j=1}^{n}\left\{B K \sum_{l=1}^{n} a_{j l}\left[x_{j}(t)-x_{l}(t)\right]+E e_{f j}(t)\right\} .
\end{aligned}
$$

For undirected graphs, we have $a_{i j}=a_{j i}$. It is easy to see that

$$
\begin{aligned}
\sum_{j=1}^{n} B K \sum_{l=1}^{n} a_{j l}\left[x_{j}(t)-x_{j}(t)\right]= & \sum_{j=1}^{n} B K \sum_{l=1}^{n} a_{j l} x_{j}(t) \\
& -\sum_{j=1}^{n} B K \sum_{l=1}^{n} a_{j l} x_{l}(t) \\
= & \sum_{j=1}^{n} B K \sum_{l=1}^{n} a_{j l} x_{j}(t) \\
& -\sum_{l=1}^{n} B K \sum_{j=1}^{n} a_{l j} x_{j}(t)=0
\end{aligned}
$$

which, together with (9), leads to

$$
\begin{aligned}
\dot{e}_{i}(t)= & A e_{i}(t)+B K \sum_{j=1}^{n} a_{i j}\left[e_{i}(t)-e_{j}(t)\right] \\
& +E\left\{e_{f i}(t)-\frac{1}{n} \sum_{j=1}^{n} e_{f j}(t)\right\} .
\end{aligned}
$$

Define $e(t) \triangleq\left[\begin{array}{llll}e_{1}^{T}(t) & \cdots & e_{n}^{T}(t)\end{array}\right]^{T}$ and $e_{f}(t) \triangleq$ $\left[\begin{array}{lll}e_{f 1}^{T}(t) & \cdots & e_{f n}^{T}(t)\end{array}\right]^{T}$. Equation (11) can be written in a compact form as

$$
\dot{e}(t)=\left(I_{N} \otimes A+\mathscr{L} \otimes B K\right) e(t)+(H \otimes E) e_{f}(t),
$$

where

$$
H=\left[\begin{array}{cccc}
\frac{n-1}{n} & -\frac{1}{n} & \cdots & -\frac{1}{n} \\
-\frac{1}{n} & \frac{n-1}{n} & \cdots & -\frac{1}{n} \\
\vdots & \vdots & \ddots & \vdots \\
-\frac{1}{n} & -\frac{1}{n} & \cdots & \frac{n-1}{n}
\end{array}\right]
$$

It can be seen that $H=I_{n}-\mathbf{1}_{n} \mathbf{1}^{T} / n, H^{2}=H, \mathbf{1}_{n}^{T} H=\mathbf{0}_{n}^{T}$, $H \mathbf{1}_{n}=\mathbf{0}_{n}$, and $H \mathscr{L}=\mathscr{L} H=\mathscr{L}$. Before giving the main results of this paper, the following lemma is needed.

Lemma 7 (see [10]). Let $H$ and $\mathscr{L}$ be matrices previously defined; the following statements hold.
(1) The eigenvalues of $H$ are 1 with multiplicity $n-1$ and 0 with multiplicity 1 . The vectors $\mathbf{1}_{n}^{T}$ and $\mathbf{1}_{n}$ are the left and right eigenvectors of $H$ associated with zero eigenvalue, respectively.

(2) There exists an orthogonal matrix $U \in \mathbb{R}^{n \times n}$ with last column $\mathbf{1}_{n} / \sqrt{n}$, such that

$U^{T} H U=\left[\begin{array}{cc}I_{n-1} & \mathbf{0}_{n-1} \\ * & 0\end{array}\right], \quad U^{T} \mathscr{L} U=\left[\begin{array}{cc}\Delta & \mathbf{0}_{n-1} \\ * & 0\end{array}\right]$.

Next, we give the main results of this paper.

Theorem 8. Suppose the undirected graph $\mathscr{G}$ is connected, and the nonzero eigenvalues of $\mathscr{L}$ are $\lambda_{2} \leq \cdots \leq \lambda_{n}$. Using protocol (6), with the fault estimator (4), consensus errors $\left\{e_{i}(t): i=\right.$ $1, \ldots, n\}$ will converge to a small ball around the origin if there exists a symmetric positive definite matrix $X$ such that the following LMI holds:

$$
A X+X A^{T}-\lambda_{2} B B^{T}<0
$$

and $K$ is chosen as $K=-B^{T} X^{-1}$.

Proof. Suppose that the communication graph is connected; the nonzero eigenvalues of $\mathscr{L}$ are $\lambda_{2} \leq \cdots \leq \lambda_{n}$ and there exists a symmetric positive definite matrix $X$ such that (15) holds. Let $Q=X^{-1}$; we have

$$
\mathrm{Q} A+A^{T} \mathrm{Q}-\lambda_{2} \mathrm{QBB} B^{T} \mathrm{Q}<0 .
$$

Define

$$
\begin{aligned}
e_{x}(t) & \triangleq\left[\begin{array}{lll}
e_{x 1}^{T}(t) & \cdots & e_{x n}^{T}(t)
\end{array}\right]^{T}, \\
f(t) & \triangleq\left[\begin{array}{lll}
f_{1}^{T}(t) & \cdots & f_{n}^{T}(t)
\end{array}\right]^{T}, \\
\dot{f}(t) & \triangleq\left[\begin{array}{lll}
\dot{f}_{1}^{T}(t) & \cdots & \dot{f}_{n}^{T}(t)
\end{array}\right]^{T},
\end{aligned}
$$

where $e_{x i}(t)=x_{i}(t)-\widehat{x}_{i}(t)$. From (1), (2), and (4), we have that

$$
\begin{aligned}
& \dot{e}_{x i}(t)=(A-K C) e_{x i}(t)+E e_{f i}(t), \\
& \dot{e}_{f i}(t)=\dot{f}_{i}(t)-\Gamma R C e_{x i}(t)+\sigma \Gamma e_{f i}(t)-\sigma \Gamma f_{i}(t) .
\end{aligned}
$$

Equation (18) can be rewritten in a compact form as

$$
\begin{aligned}
\dot{e}_{x}(t)= & {\left[I_{n} \otimes(A-K C)\right] e_{x}(t)+\left(I_{n} \otimes E\right) e_{f}(t), } \\
\dot{e}_{f}(t)= & \dot{f}(t)-\left(I_{n} \otimes \Gamma R C\right) e_{x}(t)+\left(I_{n} \otimes \sigma \Gamma\right) e_{f}(t) \\
& -\left(I_{n} \otimes \sigma \Gamma\right) f(t) .
\end{aligned}
$$

Consider the following Lyapunov function:

$$
\begin{aligned}
& V(t)=V_{1}(t)+\gamma V_{2}(t)+\gamma V_{3}(t), \\
& V_{1}(t)=e^{T}(t)\left(I_{n} \otimes Q\right) e(t), \\
& V_{2}(t)=e_{x}^{T}(t)(H \otimes P) e_{x}(t), \\
& V_{3}(t)=e_{f}^{T}(t)\left(H \otimes \Gamma^{-1}\right) e_{f}(t),
\end{aligned}
$$


where $P$ is defined in Lemma 5. It follows from (12) that

$$
\begin{gathered}
\dot{V}_{1}(t)=e^{T}(t)\left\{I_{n} \otimes\left(Q A+A^{T} Q\right)+\mathscr{L}\right. \\
\left.\otimes\left(Q B K+K^{T} B^{T} Q\right)\right\} e(t) \\
+2 e^{T}(t)(H \otimes Q E) e_{f}(t)
\end{gathered}
$$

From (19) we have

$$
\begin{array}{r}
\dot{V}_{2}(t)=e_{x}^{T}(t)\left\{H \otimes \Pi_{1}\right\} e_{x}(t)+2 e_{x}^{T}(t)(H \otimes P E) e_{f}(t), \\
\dot{V}_{3}(t)=-2 e_{f}^{T}(t)\left[(H \otimes R C) e_{x}(t)+\left(H \otimes \sigma I_{m}\right) e_{f}(t)\right. \\
\left.+\left(H \otimes \Gamma^{-1}\right) \dot{f}(t)-\left(H \otimes \sigma I_{m}\right) f(t)\right] .
\end{array}
$$

Notice that $P E=C R$ and

$$
\begin{aligned}
-2 e_{f}^{T}(t)\left(H \otimes \Gamma^{-1}\right) \dot{f}(t) \leq & e_{f}^{T}(t)\left[H \otimes \lambda_{\max }\left(\Gamma^{-1}\right) I_{m}\right] e_{f}(t) \\
& +\dot{f}^{T}(t)\left[H \otimes \lambda_{\max }\left(\Gamma^{-1}\right) I_{m}\right] \\
& \times \dot{f}(t) \\
-2 e_{f}^{T}(t)\left(H \otimes \sigma I_{m}\right) f(t) \leq & e_{f}^{T}(t)\left[H \otimes \sigma I_{m}\right] e_{f}(t) \\
& +f^{T}(t)\left[H \otimes \sigma I_{m}\right] f(t) ;
\end{aligned}
$$

it follows that

$$
\begin{aligned}
\dot{V}_{2}(t)+\dot{V}_{3}(t) \leq & e_{x}^{T}(t)\left\{H \otimes \Pi_{1}\right\} e_{x}(t) \\
& -e_{f}^{T}(t)\left[H \otimes\left(\sigma I_{m}-\Gamma^{-1}\right)\right] e_{f}(t) \\
& +\dot{f}^{T}(t)\left[H \otimes \lambda_{\max }\left(\Gamma^{-1}\right) I_{m}\right] \dot{f}(t) \\
& +f^{T}(t)\left[H \otimes \sigma I_{m}\right] f(t) .
\end{aligned}
$$

Let $U=\left[\begin{array}{ll}U_{1} & \mathbf{1}_{n} / \sqrt{n}\end{array}\right]$; we have $U_{1}^{T} H U_{1}=I_{N-1}$ and $U_{1}^{T} \mathscr{L} U_{1}=$ $\Delta$, where $U$ is defined in Lemma 7. Define

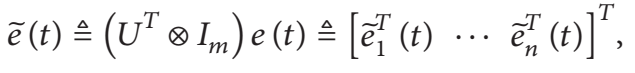

$$
\begin{aligned}
& \tilde{e}_{x}(t) \triangleq\left(\begin{array}{lll}
U^{T} \otimes I_{m}
\end{array}\right) e_{x}(t) \triangleq\left[\begin{array}{lll}
\tilde{e}_{x 1}^{T}(t) & \cdots & \tilde{e}_{x n}^{T}(t)
\end{array}\right]^{T}, \\
& \widetilde{e}_{f}(t) \triangleq\left(\begin{array}{lll}
U^{T} \otimes I_{m}
\end{array}\right) e_{f}(t) \triangleq\left[\begin{array}{lll}
\tilde{e}_{f 1}^{T}(t) & \cdots & \tilde{e}_{f n}^{T}(t)
\end{array}\right]^{T}, \\
& \tilde{f}(t) \triangleq\left(\begin{array}{lll}
U^{T} \otimes I_{m}
\end{array}\right) f(t) \triangleq\left[\begin{array}{lll}
\tilde{f}_{1}^{T}(t) & \cdots & \tilde{f}_{n}^{T}(t)
\end{array}\right]^{T},
\end{aligned}
$$

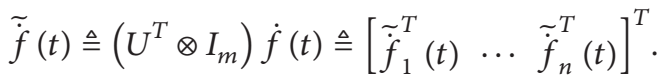

By the definition of $e(t)$ we know that $e(t)=\left(H \otimes I_{m}\right) x(t)$, where $x(t)=\left[\begin{array}{llll}x_{1}^{T}(t) & \cdots & x_{n}^{T}(t)\end{array}\right]^{T}$. It follows that

$$
\widetilde{e}_{n}(t)=\left(\frac{\mathbf{1}_{n}^{T}}{\sqrt{n}} \otimes I_{m}\right)\left(H \otimes I_{m}\right) x(t)=\mathbf{0}_{n m} .
$$

From (21) we have

$$
\begin{aligned}
& \dot{V}_{1}(t)=\tilde{e}^{T}(t)\left(U^{T} \otimes I_{m}\right)\left\{I_{n} \otimes\left(Q A+A^{T} Q\right)\right. \\
& \left.+\mathscr{L} \otimes\left(Q B K+K^{T} B^{T} Q\right)\right\} \\
& \times\left(U \otimes I_{m}\right) \tilde{e}(t) \\
& +\widetilde{e}^{T}(t)\left(U^{T} \otimes I_{m}\right)(H \otimes Q E)\left(U \otimes I_{m}\right) \tilde{e}_{f}(t) \\
& =\tilde{e}^{T}(t)\left\{I_{n} \otimes\left(Q A+A^{T} Q\right)\right. \\
& \left.+\left[\begin{array}{cc}
\Delta & \mathbf{0}_{n-1} \\
* & 0
\end{array}\right] \otimes\left(Q B K+K^{T} B^{T} Q\right)\right\} \widetilde{e}(t) \\
& +\widetilde{e}^{T}(t)\left(\left[\begin{array}{cc}
I_{n-1} & \mathbf{0}_{n-1} \\
* & 0
\end{array}\right] \otimes Q\right) \widetilde{e}_{f}(t) \\
& \leq \sum_{i=1}^{n-1}\left[\tilde { e } _ { i } ^ { T } ( t ) \left(\mathrm{Q} A+A^{T} \mathrm{Q}\right.\right. \\
& \left.-\lambda_{\min }(\Delta) Q B B^{T} Q\right) \widetilde{e}_{i}(t) \\
& \left.+\widetilde{e}_{i}^{T}(t) Q E \widetilde{e}_{f i}(t)\right] \text {. }
\end{aligned}
$$

Similarly, from (24) we have

$$
\begin{aligned}
\dot{V}_{2}(t)+\dot{V}_{3}(t) \leq \sum_{i=1}^{n-1}[ & \tilde{e}_{x i}^{T}(t) \Pi_{1} \widetilde{e}_{x i}(t) \\
& -\widetilde{e}_{f i}^{T}(t)\left(\sigma I_{m}-\Gamma^{-1}\right) \widetilde{e}_{f i}(t) \\
& +\dot{\tilde{f}}_{i}^{T}(t) \lambda_{\max }\left(\Gamma^{-1}\right) \dot{\tilde{f}}_{i}(t) \\
& \left.+\sigma I_{m} \widetilde{f}_{i}^{T}(t) \widetilde{f}_{i}(t)\right] .
\end{aligned}
$$

It then follows that

$$
\begin{gathered}
\dot{V}(t) \leq \sum_{i=1}^{n-1}\left\{\left[\tilde{e}_{i}^{T}(t) \Pi_{2} \widetilde{e}_{i}(t)+2 \widetilde{e}_{i}^{T}(t) Q E \widetilde{e}_{f i}(t)\right]\right. \\
+\gamma\left[\widetilde{e}_{x i}^{T}(t) \Pi_{1} \widetilde{e}_{x i}(t)-\widetilde{e}_{f i}^{T}(t)\left(\sigma I_{m}-\Gamma^{-1}\right) \widetilde{e}_{f i}(t)\right. \\
+\dot{\tilde{f}}_{i}^{T}(t) \lambda_{\max }\left(\Gamma^{-1}\right) \dot{\tilde{f}}_{i}(t) \\
\left.\left.+\sigma I_{m} \tilde{f}_{i}^{T}(t) \tilde{f}_{i}(t)\right]\right\},
\end{gathered}
$$

where $\Pi_{2}=Q A+A^{T} Q-\lambda_{\min }(\Delta) Q B B^{T} Q$.

Notice that $f_{i}(t)$ and $\dot{f}_{i}(t)$ are bounded, for $i=1, \ldots, n$. It is easy to see that $\dot{\tilde{f}}_{i}^{T}(t) \lambda_{\text {max }}\left(\Gamma^{-1}\right) \dot{\tilde{f}}_{i}(t)+\sigma \widetilde{f}_{i}^{T}(t) \tilde{f}_{i}(t)$ is bounded, and the bound is determined by $\sigma$ and $\Gamma$. We 
assume that $\left|\dot{\widetilde{f}}_{i}^{T}(t) \lambda_{\text {max }}\left(\Gamma^{-1}\right) \dot{\widetilde{f}}_{i}(t)+\sigma \widetilde{f}_{i}^{T}(t) \widetilde{f}_{i}(t)\right|<\beta(\sigma, \Gamma)$. Equation (29) can be rewritten as

$$
\begin{gathered}
\dot{V}(t) \leq \sum_{i=1}^{n-1}\left\{\left[\tilde{e}_{i}^{T}(t) \Pi_{1} \widetilde{e}_{i}(t)+2 \widetilde{e}_{i}^{T}(t) Q E \widetilde{e}_{f i}(t)\right]\right. \\
+\gamma\left[\tilde{e}_{x i}^{T}(t) \Pi_{2} \widetilde{e}_{x i}(t)\right. \\
-\tilde{e}_{f i}^{T}(t)\left(\sigma I_{m}-\Gamma^{-1}\right) \tilde{e}_{f i}(t) \\
+\gamma \beta(\sigma, \Gamma)]\} \\
=\sum_{i=1}^{n-1}\left[\xi_{i}^{T}(t) \Omega \xi_{i}(t)+\gamma \beta(\sigma, \Gamma)\right]
\end{gathered}
$$

where $\xi_{i}(t) \triangleq\left[\begin{array}{lll}\widetilde{e}_{i}^{T}(t) & \widetilde{e}_{x i}^{T}(t) & \widetilde{e}_{f i}^{T}(t)\end{array}\right]^{T}$ and

$$
\Omega=\left[\begin{array}{ccc}
\Pi_{2} & 0 & Q E \\
0 & \gamma \Pi_{1} & 0 \\
E^{T} Q & 0 & \gamma\left(\Gamma^{-1}-\sigma I_{m}\right)
\end{array}\right]
$$

It is easy to see that $\lambda_{\text {min }}(\Delta)=\lambda_{2}$. From the assumption of the theorem, we know that $\Pi_{2}<0$. By the definition of $\Pi_{1}$, we know that $\Pi_{1}<0$. It is easy to see that $\Omega<0$ if $\gamma$ is small enough. From (30) we know that $\dot{V}(t) \leq 0$ if

$$
\xi_{i}^{T}(t) \Omega \xi_{i}(t)+\gamma \beta(\sigma, \Gamma) \leq 0
$$

which implies that $\xi_{i}(t)$ will converge into $S_{1}=\left\{\xi_{i}\right.$ : $\left.\left|\xi_{i}^{T}(t) \Omega \xi_{i}(t)\right| \leq \gamma \beta(\sigma, \Gamma)\right\}$, yielding that $\left\{e_{i}(t): i=1, \ldots, n\right\}$ will converge to a small ball around the origin. The proof is completed.

Remark 9. Theorem 8 shows that $e_{i}(t)$ can converge to a small ball surrounding the origin. From the proof, we know that this set is determined by $\sigma$ and $\Gamma$. Since $\sigma$ and $\Gamma$ can be selected freely, this ball can be chosen arbitrarily small. However, if $\sigma$ is chosen too small, $e_{i}(t)$ may converge very slowly. To overcome this problem, dynamically changing $\sigma$ and $\Gamma$ can be used in the practice. This is out of the scope of this paper and will be considered in our future research.

\section{A Numerical Example}

Consider a multi-agent system consisting of 4 agents with

$$
\begin{aligned}
& A=\left[\begin{array}{ll}
0 & 1 \\
0 & 0
\end{array}\right], \\
& B=E=\left[\begin{array}{l}
0 \\
1
\end{array}\right], \\
& C=\left[\begin{array}{ll}
1 & 0 \\
0 & 1
\end{array}\right] ;
\end{aligned}
$$

$$
1 \longleftrightarrow 2 \longleftrightarrow 3 \longleftrightarrow 4
$$

FIGURE 1: The network topology associated with agents 1 to 4 .

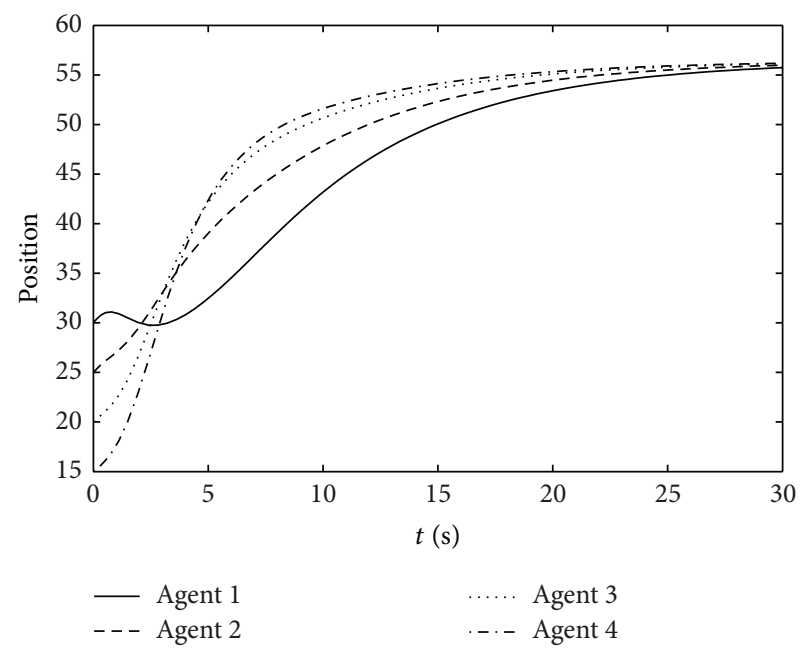

FIGURE 2: Position responses of agents 1-4.

$f_{1}(t)=f_{2}(t)=f_{3}(t) \equiv 0, f_{4}(t)=0$ for $t<1 s$, and $f_{4}(t)=0.2$ for $t \geq 1 \mathrm{~s}$. The communication topology is given in Figure 1 , with the following Laplacian matrix:

$$
\mathscr{L}=\left[\begin{array}{cccc}
1 & -1 & 0 & 0 \\
-1 & 2 & -1 & 0 \\
0 & -1 & 2 & -1 \\
0 & 0 & 1 & -1
\end{array}\right]
$$

The eigenvalues of $\mathscr{L}$ are $0,0.5858,2$, and 3.4142. According to Theorem $8, K$ can be chosen as

$$
K=\left[\begin{array}{ll}
-0.4699 & -2.5507
\end{array}\right]
$$

and parameters in the estimator (4) can be chosen as $\sigma=1$, $\Gamma=1$, and

$$
R=\left[\begin{array}{c}
0 \\
-1.3094
\end{array}\right]
$$

Figures 2 and 3 show, respectively, the position and velocity responses of nodes 1-4. It can be seen that consensus can be achieved in this case.

\section{Conclusions and Future Work}

The fault-tolerant consensus problem for multi-agent systems with actuator faults was considered. A fault estimator based consensus protocol is provided, together with a sufficient condition under which the consensus can be achieved. It is proved that consensus errors of all agents can converge to a small set around the origin. The numerical example confirmed the proposed theoretical results. In practice, many systems have stochastic Markovian jumping dynamics [27-32]. Future research efforts will be devoted to the fault tolerant consensus problem of multi-agent systems with stochastic Markovian jumping dynamics. 


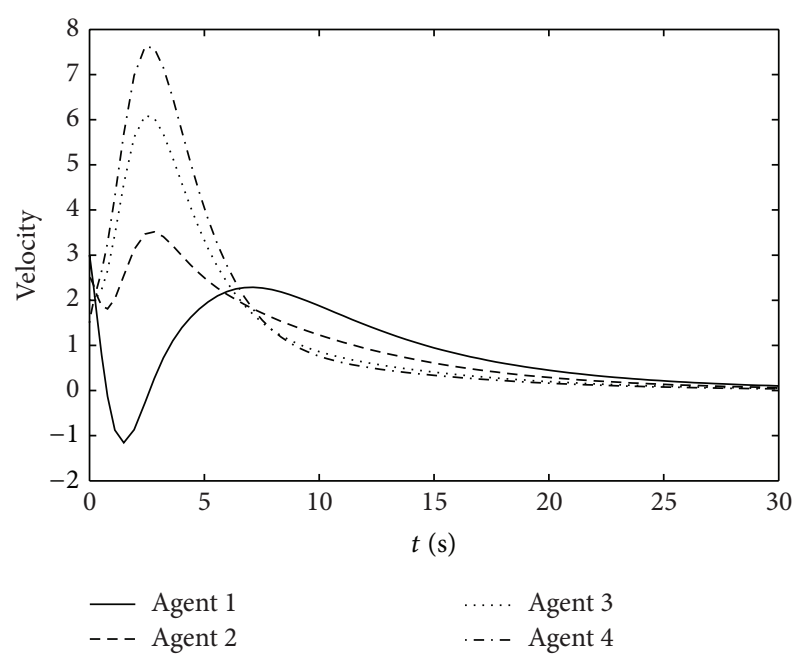

Figure 3: Velocity responses of agents 1-4.

\section{Acknowledgments}

This study was supported by National Natural Science Foundation of China under Grants 61203024, 61100116, 61374063, and 61304249, Natural Science Fundamental Research Project of Jiangsu Colleges and Universities under Grant 12KJB120001, and the Natural Science Foundation of Jiangsu Province of China under Grant BK2011492.

\section{References}

[1] R. Olfati-Saber and R. M. Murray, "Consensus problems in networks of agents with switching topology and time-delays," IEEE Transactions on Automatic Control, vol. 49, no. 9, pp. 1520-1533, 2004.

[2] W. Ren and R. W. Beard, "Consensus seeking in multiagent systems under dynamically changing interaction topologies," IEEE Transactions on Automatic Control, vol. 50, no. 5, pp. 655-661, 2005.

[3] W. Ren, "Multi-vehicle consensus with a time-varying reference state," Systems and Control Letters, vol. 56, no. 7-8, pp. 474-483, 2007.

[4] W. Yu, G. Chen, and M. Cao, "Some necessary and sufficient conditions for second-order consensus in multi-agent dynamical systems," Automatica, vol. 46, no. 6, pp. 1089-1095, 2010.

[5] H. Zhao, S. Xu, D. Yuan, J. Lu, and Y. Zou, "Minimum communication cost consensus in multi-agent systems with Markov chain patterns," IET Control Theory and Applications, vol. 5, no. 1, pp. 63-68, 2011.

[6] H. Zhao, W. Ren, D. Yuan, and J. Chen, "Distributed discretetime coordinated tracking with Markovian switching topologies," Systems \& Control Letters, vol. 61, pp. 766-772, 2012.

[7] D. Yuan, S. Xu, H. Zhao, and Y. Chu, "Accelerating distributed average consensus by exploring the information of secondorder neighbors," Physics Letters A, vol. 374 , no. 24, pp. $2438-$ 2445, 2010.

[8] D. Yuan, S. Xu, H. Zhao, and Y. Chu, "Distributed average consensus via gossip algorithm with real-valued and quantized data for $0<q<1$," Systems and Control Letters, vol. 59, no. 9, pp. 536-542, 2010.
[9] S. Liu, T. Li, and L. Xie, "Distributed consensus for multiagent systems with communication delays and limited data rate," SIAM Journal on Control and Optimization, vol. 49, no. 6, pp. 2239-2262, 2011.

[10] P. Lin, Y. Jia, and L. Li, "Distributed robust $H_{\infty}$ consensus control in directed networks of agents with time-delay," Systems and Control Letters, vol. 57, no. 8, pp. 643-653, 2008.

[11] Z. Li, Z. Duan, G. Chen, and L. Huang, "Consensus of multiagent systems and synchronization of complex networks: a unified viewpoint," IEEE Transactions on Circuits and Systems I, vol. 57, pp. 213-224, 2010.

[12] G. Wen, G. Hu, W. Yu, J. Cao, and G. Chen, "Consensus tracking for higher-order multi-agent systems with switching directed topologies and occasionally missing control inputs," Systems \& Control Letters, vol. 62, pp. 1151-1158, 2013.

[13] R. W. Beard, J. Lawton, and F. Y. Hadaegh, "A coordination architecture for spacecraft formation control," IEEE Transactions on Control Systems Technology, vol. 9, no. 6, pp. 777-790, 2001.

[14] B. Jiang, M. Staroswiecki, and V. Cocquempot, "Cooperative target tracking control of multiple robots," IEEE Transactions on Industrial Electronics, vol. 59, no. 8, pp. 3232-3240, 2012.

[15] J. A. Fax and R. M. Murray, "Information flow and cooperative control of vehicle formations," IEEE Transactions on Automatic Control, vol. 49, no. 9, pp. 1465-1476, 2004.

[16] R. Olfati-Saber and J. S. Shamma, "Consensus filters for sensor networks and distributed sensor fusion," in Proceedings of the 44th IEEE Conference on Decision and Control, and the European Control Conference (CDC-ECC '05), pp. 6698-6703, Seville, Spain, December 2005.

[17] H. Su, X. Wang, and Z. Lin, "Flocking of multi-agents with a virtual leader," IEEE Transactions on Automatic Control, vol. 54, no. 2, pp. 293-307, 2009.

[18] A. Bidram, A. Davoudi, F. L. Lewis, and Z. Qu, "Secondary control of microgrids based on distributed cooperative control of multi-agent systems," IET Control Theory and Applications, vol. 7, pp. 822-831, 2013.

[19] R. Olfati-Saber, J. A. Fax, and R. M. Murray, "Consensus and cooperation in networked multi-agent systems," Proceedings of the IEEE, vol. 95, no. 1, pp. 215-233, 2007.

[20] B. Jiang, M. Staroswiecki, and V. Cocquempot, "Fault accommodation for nonlinear dynamic systems," IEEE Transactions on Automatic Control, vol. 51, no. 9, pp. 1578-1583, 2006.

[21] S. X. Ding, Model-Based Fault Diagnosis Techniques: Design Schemes, Springer, Berlin, Germany, 2008.

[22] H. Shen, X. Song, and Z. Wang, "Robust fault tolerant control of uncertain fractional-order systems against actuator faults," IET Control Theory \& Applications, vol. 7, pp. 1233-1241, 2013.

[23] F. Pasqualetti, A. Bicchi, and F. Bullo, "Consensus computation in unreliable networks: a system theoretic approach," IEEE Transactions on Automatic Control, vol. 57, no. 1, pp. 90-104, 2012.

[24] I. Shames, A. M. H. Teixeira, H. Sandberg, and K. H. Johansson, "Distributed fault detection for interconnected second-order systems," Automatica, vol. 47, no. 12, pp. 2757-2764, 2011.

[25] H. Yang, M. Staroswiecki, B. Jiang, and J. Liu, "Fault tolerant cooperative control for a class of nonlinear multi-agent systems," Systems and Control Letters, vol. 60, no. 4, pp. 271-277, 2011.

[26] H. LeBlanc, H. Zhang, X. Koutsoukos, and S. Sundaram, "Resilient asymptotic consensus in robust networks," IEEE 
Journal on Selected Areas in Communications, vol. 31, pp. 766781, 2013.

[27] H. Shen, S. Xu, J. Lu, and J. Zhou, "Passivity-based control for uncertain stochastic jumping systems with mode-dependent round-trip time delays," Journal of the Franklin Institute, vol. 349, no. 5, pp. 1665-1680, 2012.

[28] S. He and F. Liu, "Adaptive observer-based fault estimation for stochastic Markovian jumping systems," Abstract and Applied Analysis, vol. 2013, Article ID 176419, 11 pages, 2013.

[29] S. He and F. Liu, "Robust stabilization of stochastic Markovian jumping systems via proportional-integral control," Signal Processing, vol. 91, no. 11, pp. 2478-2486, 2011.

[30] Z. Wu, P. Shi, H. Su, and J. Chu, "Passivity analysis for discretetime stochastic markovian jump neural networks with mixed time delays," IEEE Transactions on Neural Networks, vol. 22, no. 10, pp. 1566-1575, 2011.

[31] Z. Wu, P. Shi, H. Su, and J. Chu, "Asynchronous 12-11 filtering for discrete-time stochastic markov jump systems with randomly occurred sensor nonlinearities," Automatica. In press.

[32] B. Zhang, W. Zheng, and S. Xu, "Filtering of Markovian jump delay systems based on a new performance index," IEEE Transactions on Circuits and Systems I, vol. 60, pp. 1250-1263, 2013. 


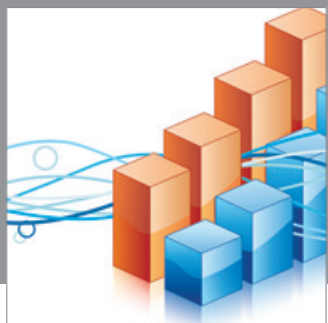

Advances in

Operations Research

mansans

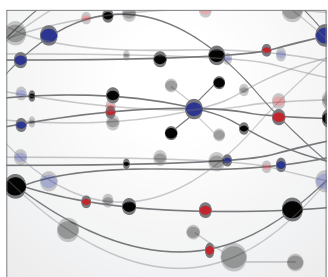

The Scientific World Journal
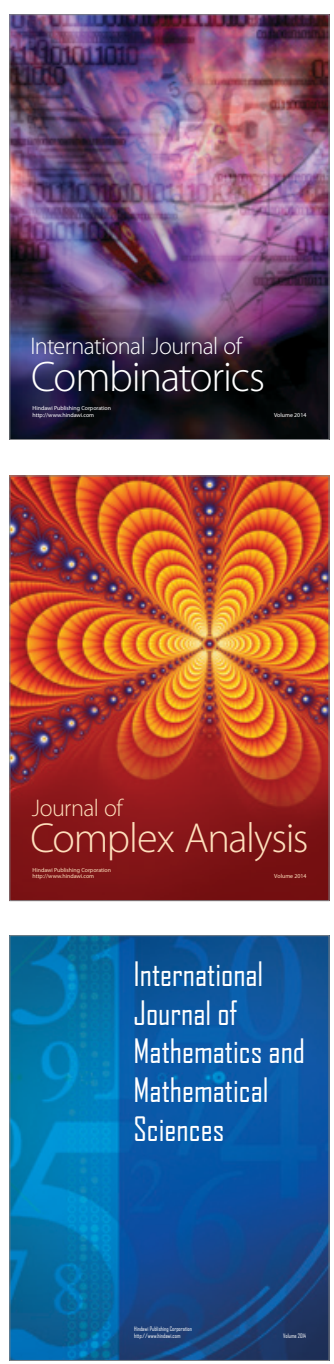
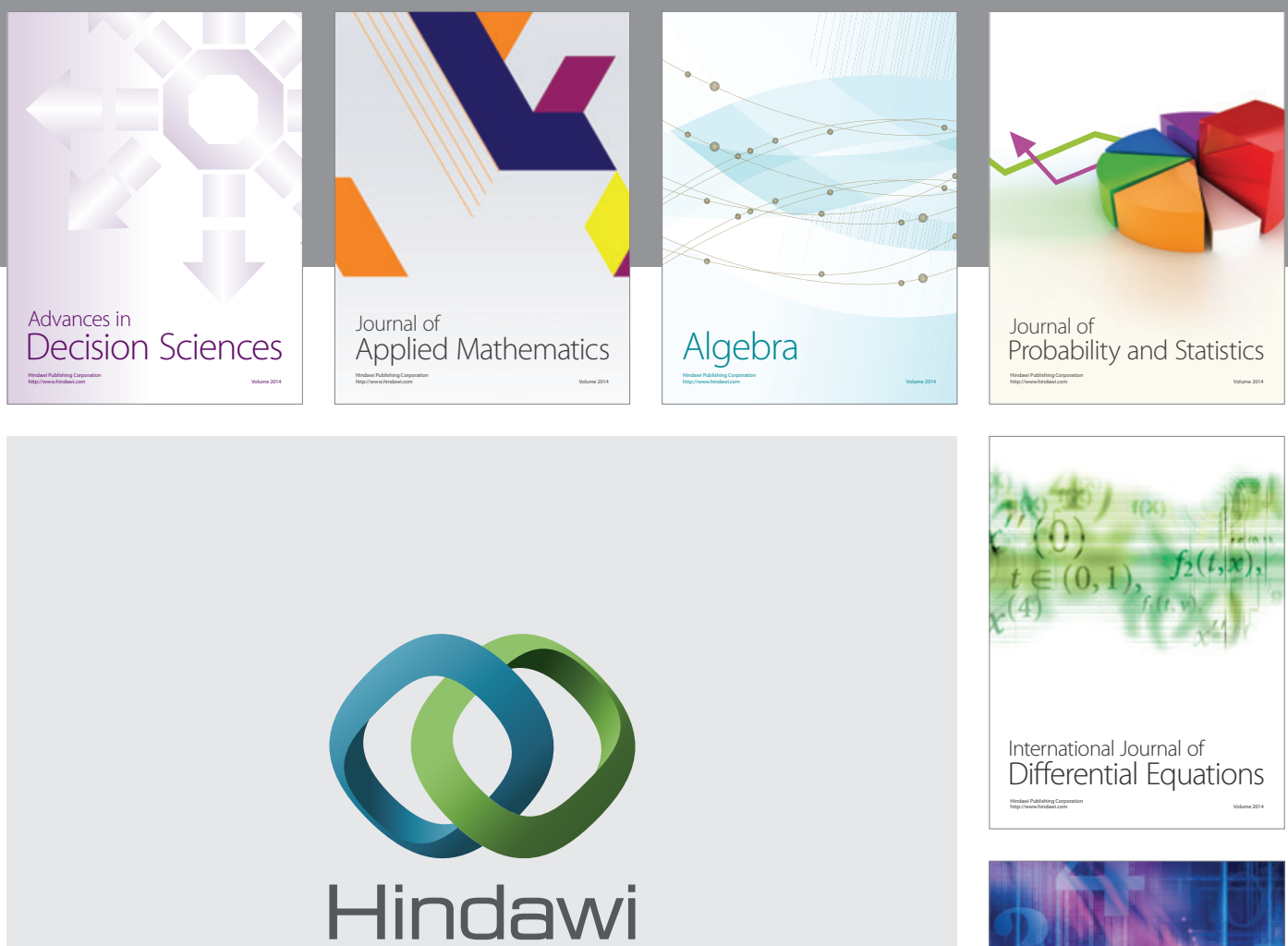

Submit your manuscripts at http://www.hindawi.com
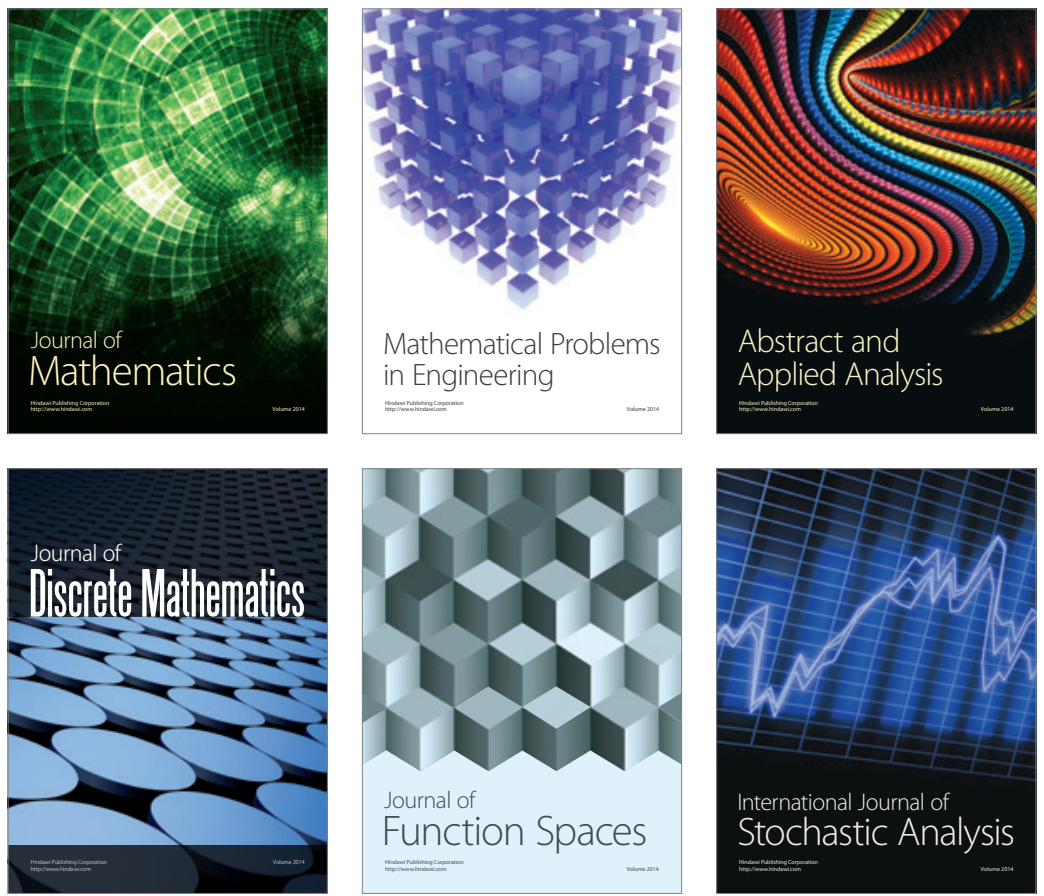

Journal of

Function Spaces

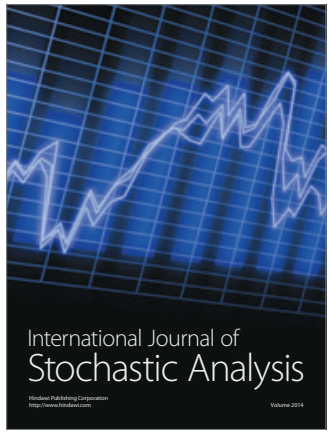

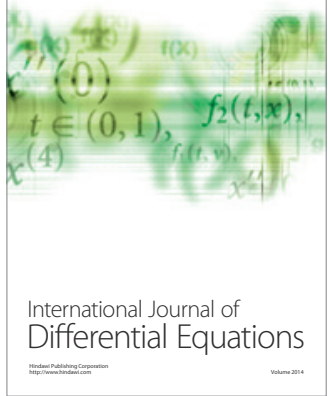
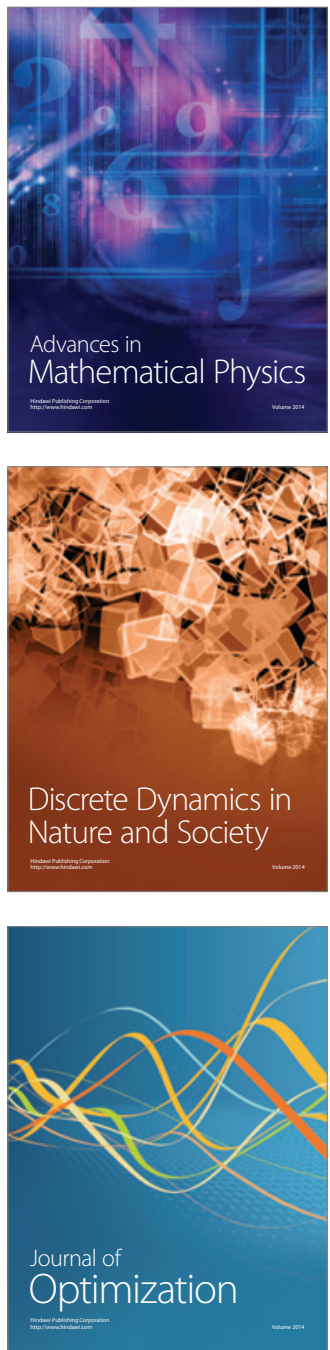